\title{
AVALIAÇÃO DE DIFERENTES PARÂMETROS REACIONAIS NA SÍNTESE DE COMPOSTOS FURÂNICOS A PARTIR DA CASCA DE ARROZ UTILIZANDO O LÍQUIDO IÔNICO [BMIM][CI]
}

Evaluation of different reactional parameters in the synthesis of furanic compounds from the rice husk using liquid [BMIM][Cl]

Evaluación de diferentes parâmetros reaccionales em la sínteses de compuestos furánicos de la cáscara de arroz utilizando líquido iónico [BMIM][Cl]

\section{Amalhia Sousa da Silva ${ }^{1}$, Elisandra Scapin ${ }^{2 *}$ \\ ${ }^{1}$ Laboratório de Química, Engenharia Ambiental, Universidade Federal do Tocantins - UFT, Palmas - TO, Brasil. \\ ${ }^{2}$ Laboratório de Química, Engenharia Ambiental, Programa de Pós-Graduação em Ciências Ambientais - Ciamb, Programa de Pós-Graduação em Biodiversidade e Biotecnologia - Bionorte, Universidade Federal do Tocantins - UFT, Palmas - TO, Brasil. \\ *Correspondência: Laboratório de Química, Universidade Federal do Tocantins, Av. NS 15, 109 Norte, Palmas, Tocantins, Brasil.CEP:77.010-090.e-mail scapin@uft.edu.br.}

\section{Artigo recebido em 13/03/2020 aprovado em 06/11/2020 publicado em 10/03/2021.}

\section{RESUMO}

Atualmente, a diminuição das atividades petroquímicas e os impactos ambientais gerados pelo consumo de combustíveis fósseis são questões fundamentais no âmbito político, social e ambiental para a maioria dos países do mundo. Diante disso a busca por matérias-primas renováveis e produtos biodegradáveis que possam ser utilizados como base na indústria química vêm ascendendo muito nos últimos anos. Neste cenário, compostos furânicos como o 5-hidroximetilfurfural (HMF) e o furfural (FF) são destaque como compostos base na indústria de química fina com alto valor agregado provenientes da biomassa. Este trabalho teve como objetivos analisar a influência das variáveis tempo e concentração de catalizador (CC) na síntese de HMF e FF a partir da matéria-prima advinda da casca de arroz, utilizando o líquido iônico (LI) [BMIM][Cl] como meio reacional. Inicialmente a casca de arroz foi seca, moída e hidrolisada em meio ácido. A síntese do HMF e FF foi realizada utilizando $0,5 \mathrm{ml}$ de acetato de etila, $1 \mathrm{~g}$ do LI e 2,5 ml de hidrolisado sob agitação constante a $140{ }^{\circ} \mathrm{C}$, variando o tempo $(0,5 ; 1,0 ; 2,0 ; 3,0$ e $3,5 \mathrm{~h})$ e a CC $(0 \%, 5 \%$, $15 \%, 25 \%, 30 \%)$. A maior concentração de $\operatorname{HMF}(3,48 \%)$ foi obtida em 3 h de reação à $5 \%$ de $\mathrm{CC}$ e a de $\mathrm{FF}$ $(41,13 \%)$ foi em 3,5h à $15 \%$ de CC. A análise estatística mostrou que o tempo e a CC têm forte significância sobre os rendimentos de HMF, porém, na síntese de FF o tempo é o fator dominante. A casca de arroz possui boa capacidade percussora para síntese de HMF e FF, e consequentemente aplicações em processos de biorrefinaria.

Palavras-chave: 5-hidroximetilfurfural; furfural; biomassa; liquido iônico. 


\begin{abstract}
Currently, the decrease in petrochemical activities and the environmental impacts generated by the consumption of fossil fuels are fundamental issues in the political, social and environmental spheres for most countries in the world. In view of this, the search for renewable raw materials and biodegradable products that can be used as a basis in the chemical industry has been on the rise in recent years. In this scenario, furan compounds such as 5-hydroxymethylfurfural (HMF) and furfural (FF) are highlighted as base compounds in the fine chemical industry with high added value from biomass. This work aimed to analyze the influence of the variables time and catalyst concentration $(C C)$ in the synthesis of $H M F$ and FF from the raw material from the rice husk, handling with ionic liquid (LI) [BMIM] [Cl] as reaction medium. Initially the rice husk was dried, ground and hydrolyzed in an acid medium. The synthesis of HMF and FF was performed using $0.5 \mathrm{ml}$ of ethyl acetate, $1 \mathrm{~g}$ of LI and $2.5 \mathrm{ml}$ of hydrolyzate under constant agitation at $140{ }^{\circ} \mathrm{C}$, varying the time $(0.5 ; 1.0 ; 2,0 ; 3.0$ and $3.5 \mathrm{~h})$ and $C C(0 \%, 5 \%, 15 \%, 25 \%, 30 \%)$. The highest concentration of HMF (3.48\%) was obtained in $3 \mathrm{~h}$ of reaction at $5 \%$ of CC and that of FF (41.13\%) was in $3.5 \mathrm{~h}$ at $15 \%$ of CC. Statistical analysis showed that time and WC have a strong significance on HMF yields, however, in FF synthesis time is the dominant factor. The rice husk has good percussion capacity for the synthesis of HMF and FF, and consequently applications in biorefinery processes.
\end{abstract}

Keywords: 5-hydroxymethylfurfural, furfural, biomass, ionic liquid.

\title{
RESUMEN
}

Actualmente, la disminución de las actividades petroquímicas y los impactos ambientales que genera el consumo de combustibles fósiles son temas fundamentales en el ámbito político, social y ambiental para la mayoría de los países del mundo. Ante esto, la búsqueda de materias primas renovables y productos biodegradables que puedan utilizarse como base en la industria química ha ido en aumento en los últimos años. En este escenario, los compuestos de furano como el 5-hidroximetilfurfural (HMF) y el furfural (FF) se destacan como compuestos base en la industria química fina con alto valor agregado proveniente de la biomasa. Este trabajo tuvo como objetivo analizar la influencia de las variables tiempo y concentración de catalizador (CC) en la síntesis de HMF y FF a partir de la materia prima de la cáscara de arroz, manipulando con líquido iónico (LI) [BMIM] [Cl] como medio de reacción. Inicialmente, la cáscara de arroz se secó, se trituró e hidrolizó en un medio ácido. La síntesis de HMF y FF se realizó utilizando 0.5 $\mathrm{ml}$ de acetato de etilo, $1 \mathrm{~g}$ de LI y $2.5 \mathrm{ml}$ de hidrolizado bajo agitación constante a $140{ }^{\circ} \mathrm{C}$, variando el tiempo $(0.5 ; 1.0 ; 2,0 ; 3.0$ y 3.5h) y CC $(0 \%, 5 \%, 15 \%, 25 \%, 30 \%)$. La mayor concentración de HMF (3,48\%) se obtuvo en 3 h de reacción al 5\% de CC y la de FF (41,13\%) fue en 3,5 h al $15 \%$ de CC. El análisis estadístico mostró que el tiempo y la WC tienen una gran importancia en los rendimientos de HMF, sin embargo, en la síntesis de FF, el tiempo es el factor dominante. La cáscara de arroz tiene buena capacidad de percusión para la síntesis de HMF y FF, y consecuentemente aplicaciones en procesos de biorrefinería.

Descriptores: 5-hidroximetilfurfural;furfural; biomasa; líquido iónico.

\section{INTRODUÇÃO}

A procura por matérias-primas renováveis e produtos biodegradáveis, vêm crescendo nos últimos anos devido à carência das fontes renováveis de energia somada à preocupação com o meio ambiente.
Desta forma, a biomassa se destaca como uma boa opção de fonte energética renovável, onde, gera baixas quantidades de poluentes, proporciona o reaproveitamento de resíduos, contém alta eficiência energética, e, uma fonte de 
energia renovável e limpa, emite menos poluentes (Cortez; Gómez; Lora, 2008).

A utilização da biomassa como produto em si, tem como grandes vantagens seu aproveitamento direto por meio da combustão de diversas formas como fonte energética, e também a redução de impactos socioambientais. Como desvantagens, seu aproveitamento apresenta eficiência reduzida, em situações isoladas, contudo, o potencial energético dependerá tanto da matéria-prima utilizada quanto da tecnologia adotada no processamento, (ANEEL, 2002).

Neste sentido, tem-se gerado discussões sobre diversas alternativas aos produtos petroquímicos, podendo ser convencionais e em sua maioria sustentáveis, com grande diversidade e potencial, visando diminuir o uso e a geração de substâncias tóxicas, o consumo mais eficiente de energia e o desenvolvimento de estratégias de mitigação de impactos ambientais, (Bastos et al., 2007).

Dentre os principais cereais produzidos e consumidos no mundo, o arroz se destaca principalmente por ser um alimento base para mais da metade da população mundial. Sua importância é evidenciada principalmente em países em desenvolvimento, tais como o Brasil, desempenhando papel estratégico em níveis econômico e social, segundo dados do IBGE (2020).

Com o desenvolvimento de produtos especializados de alto valor agregado, a Química Fina destaca-se por permitir que, a partir de substâncias estruturalmente simples, encontradas em abundância na natureza e de baixo custo, possa-se chegar a substâncias mais complexas e com propriedades de grande aplicação prática (Iris et al., 2017).
Pesquisas fundamentadas em tecnologias renováveis tem como base fontes limpas de energia, levando a minimização dos impactos no meio ambiente além de estarem relacionadas com as necessidades socioeconômicas (Wang et al., 2019).

Neste cenário, os compostos da série furânica como o 5-hidroximetilfurfural (HMF) e o furfural (FF), têm ganhado destaque em processos de conversão de biomassa, pois vários recursos de biomassa renovável aparecem como matéria-prima alternativa, permitindo uma rota química mais sustentável nos processos de síntese (Van Nguyen et al., 2016; Yu et al., 2017). Em contrapartida, a produção comercial atual de HMF e FF, interesse deste trabalho, depende predominantemente dos xaropes extraídos de culturas energéticas, o que aumenta significativamente os custos de sua produção 307 (Kläusli, 2014).

O $\mathrm{HMF}$ tem despertado significativo interesse no âmbito comercial e industrial por tratar-se de uma plataforma química de alto valor agregado nos processos de conversão, atuando como intermediário químico na fabricação de pigmentos, polímeros, cosméticos, produtos farmacêuticos, biocombustíveis líquidos, dentre outros (Azevedo, 2015).

No processo de conversão de açúcares em HMF e FF, tem ganhado destaque a utilização dos líquidos iônicos (LIs) como meio reacional devido a potencialização da produção, levando à economia de energia em comparação com os solventes convencionais (Stark, 2011; Yi et al., 2010). Igualmente, o caráter iônico dos LIs pode desempenhar um papel positivo no aprimoramento da atividade catalítica e da seletividade da reação (Olivier et al., 2010), bem 
como na estabilização das espécies reativas intermediárias da reação (Zhang et al., 2017; Hu e Yang 2014). Além do mais, o uso dos LIs permite o desenvolvimento de processos simples e eficientes devido à recuperação simplificada do solvente e do produto (Peleteiro et al., 2016).

O uso de catalisadores ácidos nos processos de síntese de HMF e FF também tem se mostrado vantajoso pois acelera a degradação da celulose. Dentre estes, os catalisadores homogêneos mostram-se mais eficientes na degradação do produto em HMF, uma vez que atum em diferentes processos (Li et al., 2014).

Diante disso, o presente trabalho teve como objetivo avaliar o aproveitamento da biomassa bruta de casca de arroz na obtenção dos compostos furânicos HMF e FF, utilizando o líquido iônico Cloreto de 1-butil-3-metilimidazól ([BMIM] $[\mathrm{Cl}])$ e o catalisador Cloreto de Cromo III hexaidratado $\left(\mathrm{CrCl}_{3} \cdot 6 \mathrm{H}_{2} \mathrm{O}\right)$.

\section{MATERIAIS E MÉTODOS OBTENÇÃO DA AMOSTRA}

A casca de arroz (CA) foi coletada foi como resíduo de uma fazenda de produção local, no município de Palmas, Tocantins, onde foi posteriormente limpa e separada manualmente.

\section{DETERMINAÇÃO DA COMPOSIÇÃO QUÍMICA DA CASCA DE ARROZ}

A análise da composição química da casca de arroz foi realizada através de prétratamentos físicos e químicos, que propiciaram a liberação dos principais componentes químicos da casca de arroz, seguido de avaliação qualitativa e quantitativa dos mesmos.
No pré-tratamento físico foi realizada a moagem da casca de arroz usando moinho de facas tipo Willey (22 mesh) (Start FT 50 Fortinox), peneiradas até o tamanho de partículas $(180-850 \mu \mathrm{m})$ e armazenadas em frascos de vidros herméticos para uso posterior. Já no pré-tratamento químico foi realizada a hidrólise ácida segundo metodologia desenvolvida por Dunning e Dallas (1949).

Depois de moída a casca de arroz foi seca em estufa a $60^{\circ} \mathrm{C}$ (SSD SolidSteel 110L), por 24 horas. Após foi pesado 2g de biomassa em um béquer de $100 \mathrm{~mL}$, então adicionou-se $10 \mathrm{ml}$ de ácido sulfúrico $\left(\mathrm{H}_{2} \mathrm{SO}_{4}\right)$ a $72 \%$. A mistura foi agitada continuamente com bastão de vidro por 7 minutos em banho maria a $50{ }^{\circ} \mathrm{C}$. Após isso adicionou-se $40 \mathrm{~mL}$ de água destilada e o conteúdo transferido para um Erlenmeyer de 250 $\mathrm{mL}$. A solução foi levada à autoclave à $121^{\circ} \mathrm{C}$ por 15 minutos. Após resfriamento, foi feita a filtragem em papel filtro previamente pesado separando a fração sólida da fração líquida. A fração líquida do hidrolisado foi usada para avaliação de carboidratos e compostos furânicos (HMF e FF), e para posterior reação com o LI. Já a fração sólida que ficou retida no papel filtro foi utilizada para analisar do teor de lignina ácida insolúvel e cinzas.

\section{DETERMINAÇÃO DO TEOR DE ÁCIDA LIGNINA INSOLÚVEL}

Para a determinação do teor de lignina insolúvel o material residual presente no papel filtro foi lavado com água destilada para a remoção do $\mathrm{H}_{2} \mathrm{SO}_{4}$, até que o mesmo ficasse com a menor quantidade de sólidos retidos possível em 
suas laterais. O papel foi então seco em estufa a $60{ }^{\circ} \mathrm{C}$ até atingir massa constante.

Utilizou-se a Equação 01 baseada nos trabalhos de Rocha (2006), para efetuar o cálculo da porcentagem de lignina ácida insolúvel, também chamada lignina Klason (KL).

$$
\% \text { lignina }=\frac{\mathrm{M}_{\mathrm{K}}-\mathrm{M}_{\mathrm{C}}}{\mathrm{M}_{\mathrm{A}}} \times 100
$$

$\mathrm{M}_{\mathrm{K}}=$ Massa da lignina insolúvel.

$\mathrm{M}_{\mathrm{C}}=$ Massa das cinzas.

$\mathrm{M}_{\mathrm{A}}=$ Massa seca da amostra.

\section{DETERMINAÇÃO DO TEOR DE CINZAS}

Para determinação do teor de cinzas, a lignina retida no papel filtro foi colocada cuidadosamente em um cadinho previamente calcinado e pesado em balança analítica.

Posteriormente o cadinho com a lignina foi levado à mufla a temperatura a $600^{\circ} \mathrm{C}$ por 4 horas. Após o resfriamento as amostras de cinzas foram pesadas e o cálculo foi realizado segundo metodologia desenvolvida por Rocha (2000) (Equação 02):

$$
\% \text { Cinzas }=\frac{\mathrm{M}_{\mathrm{C}}}{\mathrm{M}_{\mathrm{A}}} \times 100
$$

Mc: Massa das cinzas.

$\mathrm{M}_{\mathrm{A}}=$ Massa seca da amostra.

\section{DETERMINAÇÃO DO TEOR DE CELULOSE E HEMICELULOSE}

Os teores de fibra em detergente ácido (FDA) e detergente neutro (FDN) foram determinadas de acordo com Trujillo; Marichal; Carriquiry (2010). O teor de hemicelulose foi determinado pela diferença entre os valores de FDN e FDA, enquanto o teor de celulose foi obtido por diferença entre o conteúdo de hemicelulose e lignina (Rambo et al., 2017).

\section{SÍNTESE DO LIQUIDO IÔNICO CLORETO DE 1-BUTIL-3-METILIMIDAZOL}

A síntese do cloreto de 1-butil-3metilimidazol ([BMIM][Cl]) foi realizada de acordo com a metodologia de Siankevich et al., (2016), a partir da reação entre 1-clorobutano e 1metilimidazol, na razão molar de 1,1: 1, em um balão de fundo redondo sob agitação por 48 horas à temperatura de $80^{\circ} \mathrm{C}$.

\section{PLANEJAMENTO EXPERIMENTAL}

O planejamento e análise experimental foram realizados usando o programa Protimiza Experimental Design (PROTIMIZA, 2014). Com base em experimentos encontrados na literatura (Carvalho et al., 2018, Scapin et al., 2020), a 309 influência da concentração do catalisador Cloreto de Cromo III hexahidratado $\left(\mathrm{CrCl}_{3} \cdot 6 \mathrm{H}_{2} \mathrm{O}\right)$ e do tempo de reação foram avaliadas através de um delineamento composto central rotacional (DCCR) com fatorial $2^{2}$, incluindo 4 ensaios em condições axiais e 3 repetições de pontos centrais, totalizando 11 tentativas (Tabela 1). Após a triagem, foi determinada a superfície de resposta e o ponto ideal para a seletividade do FF e do HMF.

\section{SÍNTESE DE HMF E FF}

A síntese de HMF e FF foi realizada de acordo com o DCCR e adaptações da metodologia desenvolvida por Yi et al. (2011). $1 \mathrm{~g}$ do LI [BMIM][Cl] e o catalisador $\mathrm{CrCl}_{3} \cdot 6 \mathrm{H}_{2} \mathrm{O}$ (nas proporções de $0 \%, 5 \%, 15 \%, 25 \%, 30 \%$ ) foram colocados em um balão de fundo redondo em banho de óleo à $140^{\circ} \mathrm{C}$ com agitação constante 
por 15 minutos. Após foi adicionado 2,5ml de amostra hidrolisada e $0,5 \mathrm{ml}$ de acetato de etila, variando o tempo $(0,5 ; 1,0 ; 2,0 ; 3,0$ e 3,5h). Após completado o tempo de reação, o produto foi lavado três vezes com acetato de etila.

Tabela 1: Esquema da Matriz de Planejamento DCCR $2^{2}$ com 3 repetições no ponto central para a produção de HMF e FF.

\begin{tabular}{ccccccc}
\hline Ensaio & Catal. & Tempo & \multicolumn{2}{c}{ HMF } & \multicolumn{2}{c}{ FF } \\
& $(\%)$ & $(\mathrm{h})$ & $(\mathrm{ppm})$ & $(\%)$ & $(\mathrm{ppm})$ & $(\%)$ \\
\hline 1 & 5 & 1 & 36,27 & 2,23 & 151,01 & 31,21 \\
2 & 5 & 3 & 56,49 & 3,48 & 157,58 & 32,82 \\
3 & 25 & 1 & 33,50 & 2,06 & 153,12 & 31,90 \\
4 & 25 & 3 & 37,14 & 2,29 & 176,63 & 36,51 \\
5 & 15 & 0,5 & 51,72 & 3,18 & 154,33 & 31,90 \\
6 & 15 & 3,5 & 54,38 & 3,35 & 198,98 & 41,13 \\
7 & 0 & 2 & 37,36 & 2,30 & 165,80 & 34,27 \\
8 & 30 & 2 & 7,43 & 0,46 & 124,51 & 25,74 \\
9 & 15 & 2 & 31,31 & 1,93 & 151,32 & 31,28 \\
10 & 15 & 2 & 39,48 & 2,43 & 129,21 & 26,71 \\
11 & 15 & 2 & 42,52 & 2,62 & 126,74 & 26,20 \\
\hline
\end{tabular}

ANÁLISE POR ESPECTROSCOPIA NO INFRAVERMELHO COM TRANSFORMADA DE FOURIER (FT-IR)

O espectrômetro de infravermelho (FTIR CARY 630, Agilent Technologies) com faixa de varredura de 650 a $4000 \mathrm{~cm}^{-1}$, usando cristal de diamante com refletância total atenuada (ATR), com $0,4 \mathrm{~nm}$ de incremento e 32 varreduras de médias foram usadas para analisar as amostras hidrolisadas, o LI e os produtos após reação com o LI e o catalisador.

\section{ANÁliSE POR CROMATOGRAFIA LÍQUIDA DE ALTA EFICIÊNCIA}

A determinação de carboidratos, HMF e FF do hidrolisado e após a realização do DCCR, foi realizada utilizando
Cromatografia Líquida de Alta Eficiência (CLAE), usando cromatógrafo Shimadzu ${ }^{\circledR}$ (LC-10 Avp series, Kyoto, Japan). Todas as amostras foram previamente diluídas e filtradas em filtro de membrana de 0,22 $\mu \mathrm{m}$ de polivinilidenodifluoreto (PVDF). As concentrações destes compostos foram calculadas a partir de curvas de calibração obtidas de soluções padrões.

Na determinação dos teores de açúcar foi utilizado coluna Phenomenex Rezex ROAOrganic ácid $\mathrm{H}^{+}(81 \%)$, utilizando ácido $\mathrm{H}_{2} \mathrm{SO}_{4}$ (5mM) como eluente, com fluxo de $0,6 \mathrm{~mL} \mathrm{~min}^{-1}$ e detector de índice de refração modelo SPD-10A VP marca Shimadzu. O volume da amostra injetada foi de $20 \mu \mathrm{L}$.

A determinação de HMF e FF foi realizada utilizando Coluna Phenomenex Luna C18 $5 \mu \mathrm{m}(2)(250 \times 4,6 \mathrm{~mm})$ e pré-coluna Phenomenex C18 $(4 \times 3,0 \mathrm{~mm})$ preenchidos com material semelhante à coluna principal. $\mathrm{O}$ fluxo do eluente foi de $1 \mathrm{~mL} \cdot \mathrm{min}^{-1}$ a $30{ }^{\circ} \mathrm{C}$, com um tempo total de execução de 15 min. A eluição isocrática foi realizada com solução de acetonitrila/água (1:8 com 1\% de ácido acético). O detector usado foi UV (SPD-10A VP) com um comprimento de onda de $276 \mathrm{~nm}$. Os rendimentos de HMF e FF foram determinados segundo as equações (03) e (04) propostas por Cai et al. (2017), respectivamente.

$H M F(\%)=$
$\frac{H M F \text { Conc. }(p p m) x \text { Vol.de líquido }(2,5 \mathrm{~mL})) / 126}{m g \text { de celulose } / 162} \times 100$

$F F(\%)=$ $\frac{F F \text { Conc. }(p p m) x \text { vol.de líquido }(2,5 m L)) / 96}{m g \text { de hemicelulose } / 132} \times 100$ 
RESULTADOS E DISCUSSÃO

DETERMINAÇÃO DA COMPOSIÇÃO

QUÍMICA DA BIOMASSA

Como a casca de arroz é encontrada em muitas regiões do Brasil, sua composição química pode variar de acordo com a composição do solo, características climáticas e diferentes qualidades do grão. Assim, para que a biomassa possa ter utilizada em processos de biorrefinaria, é de suma importância conhecer os seus aspectos físicos e as propriedades químicas. A Tabela 2 mostra a composição química da casca de arroz.

Tabela 2: Composição química da casca de arroz.

\begin{tabular}{cc}
\hline Componentes & $\begin{array}{c}\text { Concentração (\%) } \\
\text { Arroz }\end{array}$ \\
\hline Lignina Insolúvel & 31,12 \\
Cinzas & 5,07 \\
Glicose & 1,78 \\
Frutose & 4,19 \\
HMF & 0,01 \\
FF & 0,04 \\
Celulose & 52,25 \\
Hemicelulose & 16,63 \\
\hline
\end{tabular}

De acordo com Bruun et al. (2010), para que uma biomassa possa ser utilizada como como matéria-prima na formação de bioprodutos é importante conter baixo teor de cinzas em sua composição química, o que demonstra que resultado obtido para a casca de arroz $(5,07 \%$ de cinzas) é satisfatório. Uma alta concentração no teor de cinzas pode diminuir o poder calorífico e causar perda de energia (Klautau, 2008).

Já estudos realizados por Vieira et al. (2013), a casca de arroz apresentou alto teor de matéria volátil $(82,09 \%)$ e teor de cinzas relativamente alto $(15,51 \%)$, demonstrando, que neste caso, a biomassa é adequada para geração de energia, porém deve ser analisada para escolha da tecnologia e uso final.

Segundo Foletto et al. (2005), a sílica, presente nas cinzas obtidas da queima da casca de arroz, é utilizada na elaboração de diversos materiais, em diferentes ramos industriais, tais como o da construção civil, cerâmica e de vidros.

Conforme Pouey (2006), a casca de arroz, revestimento formado durante o crescimento do grão, é um material fibroso, cuja composição química é formada principalmente de celulose (50\%), lignina (30\%) e resíduos orgânicos (20\%). Valores estes semelhantes ao encontrados neste trabalho tanto para o teor de celulose $(52,25 \%)$ quanto para lignina $(31,12 \%)$. O alto teor de lignina obtido indica que a $\mathrm{CA}$ possui bom potencial para utilização em processos de pirólise.

Os bons rendimentos de açúcares frutose e glicose também evidenciam potencial para uso em biorrefinarias e consequentemente, na síntese dos compostos furânicos HMF e FF. Segundo Bevilaqua et al (2010), a hidrólise da celulose em meio ácido leva a liberação de monômeros de glicose, que, por aquecimento se desidrata e forma $\mathrm{HMF}$.

De acordo com Yi et al. (2015), a casca de arroz além de ser abundante e renovável, pode, depois de carbonizada, ser empregada na obtenção de materiais de construção alternativos como aglomerados, revestimento ecológico, substrato, adsorventes, cimentos, etc, na produção de químicas finas e na biorrefinaria.

\section{SINTESE DO LÍQUIDO IÔNICO [BMIM][CI]}

A estrutura do LI [BMIM][Cl] foi confirmada por FT-IR usando cristal de diamante 
(do ingles Attenuated Total Reflectance - ATR) e dados da literatura (Dharaskar et al., 2013; Yassin et al., 2015) (Figura 1). Em $2924 \mathrm{~cm}^{-1} \mathrm{e} 2855 \mathrm{~cm}^{-}$

1 encontram-se os picos correspondentes ao estiramento alifático assimétrico e simétrico $(\mathrm{C}-$ H) dos grupos metila. Em $3381 \mathrm{~cm}^{-1}$ encontra-se um pico alargado atribuído ao sal quaternário de amina com cloro. O pico em $1168 \mathrm{~cm}^{-1}$ correspondem às vibrações das ligações H-C-C e H-C-N do anel imidazol. Os picos entre $1458 \mathrm{~cm}^{-}$ 1 e $1569 \mathrm{~cm}^{-1}$ correspondem às vibrações das ligações H-C-C e H-C-N do anel imidazol. A banda com comprimento de onda em $726 \mathrm{~cm}^{-1}$ é devido à vibração C-N (Scapin et al., 2020).

Figura 1 - FT-IR usando ATR do líquido iônico [BMIM][Cl].

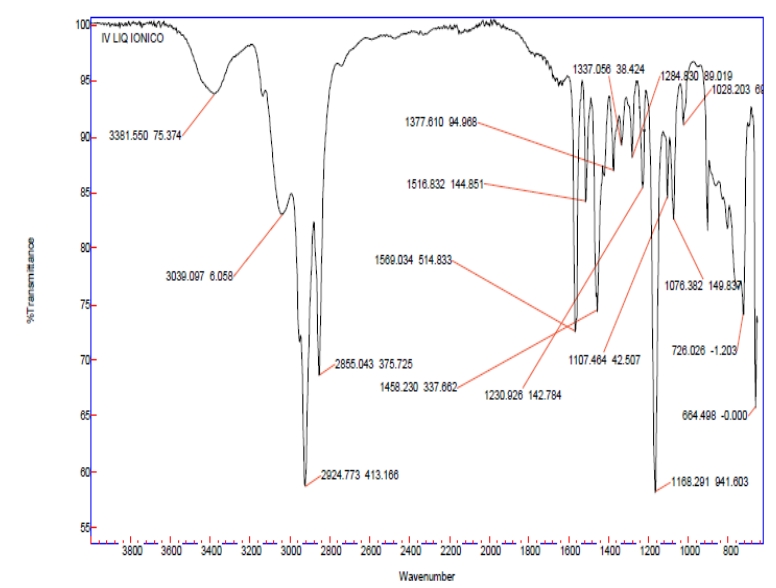

\section{SUPERFÍCIE DE RESPOSTA}

A Tabela 1 apresenta as concentrações e os rendimentos de HMF and FF obtidos após otimização experimental delineada pelo DCCR.

O maior rendimento de $\operatorname{HMF}(3,48 \%)$ foi obtido após 3 horas de reação, usando $5 \mathrm{mg}$ de catalisador (ensaio 2). O ensaio 6, após 3,5h de reação e usando $15 \mathrm{mg}$ de catalisador também levou a bons rendimentos de $\operatorname{HMF}(3,35 \%)$ e ao maior rendimento de FF, ambos utilizando o LI [BMIM] $[\mathrm{Cl}]$.
Em estudo realizado por Scapin et al., (2020), HMF foi obtido com $8,7 \%$ de rendimento após 1 hora de reação a $140{ }^{\circ} \mathrm{C}$, e o FF com $34 \%$, após 4 horas de reação a $120^{\circ} \mathrm{C}$, ambos usando o LI [BMIM][Br].

Zhang et al Zhao (2010), sintetizaram HMF e FF com 45 a $52 \%$ e 23 a $31 \%$ de rendimento, respectivamente, a partir de caule de milho, palha de arroz e madeira de pinho usando líquido iônico na presença de $\mathrm{CrCl}_{3}$.

Em trabalho desenvolvido por Wang. (2014), HMF foi obtido com até 51\% usando [BMIM][Cl], $\mathrm{CrCl}_{3} \cdot 6 \mathrm{H}_{2} \mathrm{O}$ como catalisador, a 130 ${ }^{\circ} \mathrm{C}$ por $2 \mathrm{~h}$. Os autores ressaltam que o sistema $[\mathrm{BMIM}][\mathrm{Cl}] / \mathrm{CrCl}_{3} \cdot 6 \mathrm{H}_{2} \mathrm{O} / \mathrm{HCl}$ possui alta atividade e seletividade para a desidratação da celulose em HMF.

Ainda segundo Wang et al. (2014), o uso de LIs aliado ao uso de catalisadores, como 312 desenvolvido em seu trabalho, proporciona um processo de baixo custo, favorável ao meio ambiente e com baixo consumo de energia para converter diretamente biomassa bruta em biocombustíveis e produtos químicos.

A Figura 2 mostra os diagramas de superfície de resposta obtidos a partir do planejamento executado para HMF (Figura 2a) e para o FF (Figura 2b). Pode-se observar que, para o sistema estudado, a concentração de catalisador e condições de tempo mostram um rendimento máximo de HMF no tempo de 3 horas a $5 \mathrm{mg}$ de catalisador e para FF no tempo de 3,5 horas a 15 mg de catalisador.

A análise de variância (ANOVA) aplicada para obter o modelo matemático com nível de significância $\alpha=0,05$ verificou a significância de segunda ordem para o modelo estabelecido para HMF e FF, obtendo valores de 
$\mathrm{F}_{0,05}=0,00167$ (Tabela 3) e 0,1403 (Tabela 4), respectivamente. Além disso, os valores de $\mathrm{F}$ calculado $\left(F_{\text {calc }}\right)$ foram maiores que o valor $F(p$ valor), o que valida o modelo matemático usado no experimento.

Figura 2. Superfície de resposta para (A) HMF e

(B) FF.
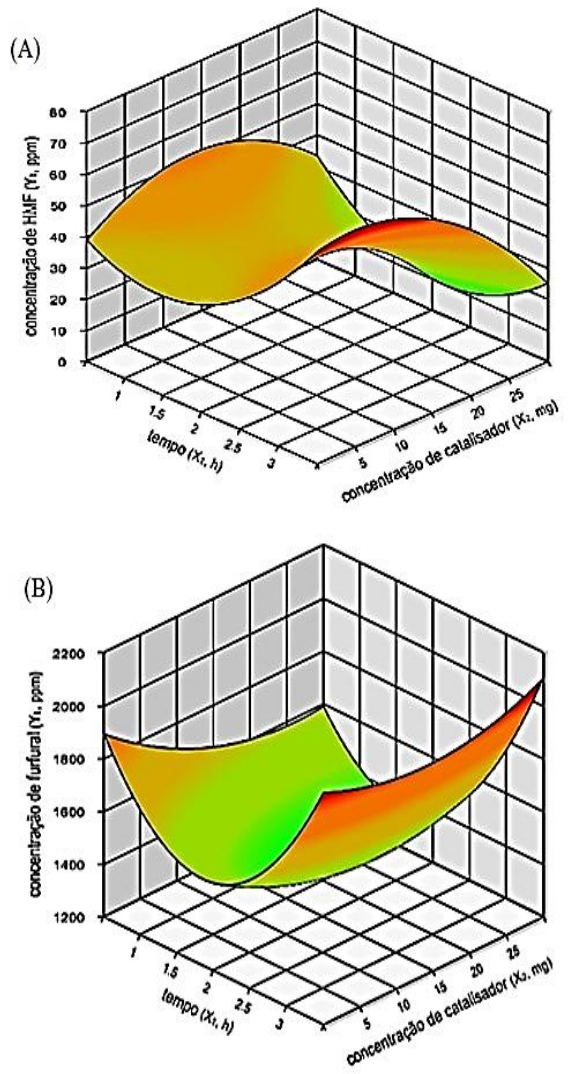

Tabela 3. ANOVA para o rendimento de HMF a partir casca de arroz.

\begin{tabular}{lccccc}
\hline Variação & $\begin{array}{c}\text { Soma dos } \\
\text { quadrados }\end{array}$ & $\begin{array}{c}\text { Graus de } \\
\text { liberdade }\end{array}$ & $\begin{array}{c}\text { Quadrado } \\
\text { médio }\end{array}$ & Fvalor & $p$-valor \\
\hline Regressão & 1628,2 & 5 & 325,6 & 8,6 & $\begin{array}{c}\mathrm{F}_{0,05}= \\
0,00167\end{array}$ \\
Resíduos & 188,4 & 5 & 37,7 & & \\
Total & 1816,6 & 10 & & & \\
\hline
\end{tabular}

Avaliando-se a relação entre o tempo e a concentração de catalisador, é possível observar que, para o HMF, o modelo utilizado apresentou alta relevância, obtendo-se coeficiente de determinação $\left(\mathrm{R}^{2}\right)$ de 89,63 . Isto mostra que o ajuste do modelo é satisfatório para o HMF. Já para o $\mathrm{FF}$, os resultados obtidos demonstraram pouca relevância, com coeficiente de determinação $\left(\mathrm{R}^{2}\right)$ de 73,78 .

Tabela 4. ANOVA para o rendimento de FF a partir da casca de arroz.

\begin{tabular}{lccccc}
\hline Variação & $\begin{array}{c}\text { Soma dos } \\
\text { quadrados }\end{array}$ & $\begin{array}{c}\text { Graus de } \\
\text { liberdade }\end{array}$ & $\begin{array}{c}\text { Quadrado } \\
\text { médio }\end{array}$ & $\mathrm{F}_{\text {valor }}$ & $p$-valor \\
\hline Regressão & 364535,4 & 5 & 72907,1 & 2,8 & $\begin{array}{c}\mathrm{F}_{0,05}= \\
0,14033\end{array}$ \\
Resíduos & 129533,0 & 5 & 25906,6 & & \\
Total & 494068,4 & 10 & & & \\
\hline
\end{tabular}

A ANOVA aplicada também demonstrou que para a produção de HMF a temperatura $\left(\mathrm{x}_{1}^{2}\right)$ e concentração de catalisador $\left(\begin{array}{lll}\mathrm{x}_{2} & \mathrm{e} & \mathrm{x}_{2}{ }^{2}\end{array}\right)$ são significativos e para o FF produção apenas a temperatura $\left(\mathrm{x}_{1}^{2}\right)$ é significativa. Isso pode ser concluído analisando o diagrama de Pareto para HMF (Figura 1a) e FF (Figura 1b).

A equação do modelo foi obtida com as variáveis tempo $\left(\mathrm{x}_{1}\right)$ e concentração de catalisador $\left(\mathrm{x}_{2}\right)$. As equações para as variáveis codificadas são descritas abaixo, onde $\mathrm{Y}_{1}$ representa $\mathrm{o}$ rendimento para o $\mathrm{HMF}$ e $\mathrm{Y}_{2} \mathrm{o}$ rendimento para o FF.

$$
\begin{aligned}
& Y_{1}=37,77+3,45 x_{1}+8,42 x_{1}{ }^{2}-8,06 x_{2}-6,91 x_{2}{ }^{2} \\
& -4,15 \mathrm{x}_{1} \mathrm{x}_{2} \\
& Y_{2}=1357,58+116,53 \mathrm{x}_{1}+202,70 \mathrm{x}_{1}{ }^{2}-46,54 \mathrm{x}_{2} \\
& +45,21 \mathrm{x}_{2}{ }^{2}+36,32 \mathrm{x}_{1} \mathrm{x}_{2}
\end{aligned}
$$


Figura 1. Diagrama de pareto dos efeitos padrões de (a) HMF e (b) FF DCCR, em que $x_{1}$ é o tempo e $\mathrm{x}_{2}$ é a Concentração de catalisador.

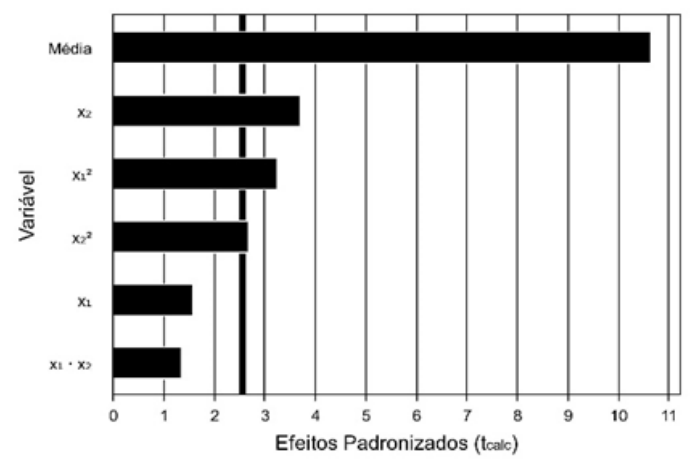

(a)

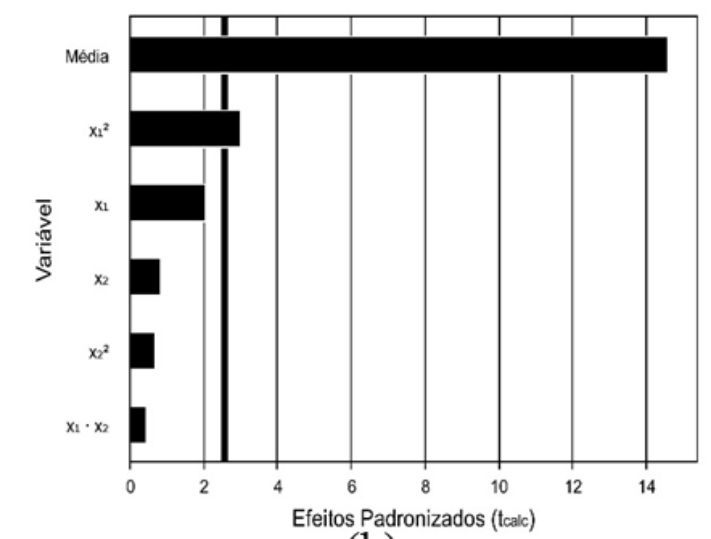

(b)

\section{CONCLUSÃO}

$\mathrm{O}$ uso de biomassa residual em processos de biorrefinaria é extremamente importante para agregar valor econômico, social e ambiental aos produtos obtidos a partir desta. Diante disso, a reutilização da casca de arroz elimina uma responsabilidade ambiental, ajuda a reduzir o custo envolvido com a produção de produtos químicos de alto valor agregado e é uma biomassa amplamente distribuída em todo o Brasil. A caracterização química mostrou que a casca de arroz pode servir como fonte segura e promissora de compostos de alto valor agregado em vários campos do setor científico e tecnológico. Outrossim, destaca-se a efetividade do líquido iônico [BIMIM][Cl] como um solvente não poluente, poder ser reutilizado ao final de cada reação e levar a obtenção de compostos furânicos HMF e FF com bons rendimentos, mostrando-se assim efetivo para este fim.

\section{AGRADECIMENTOS}

Agradecemos à universidade Federal do Tocantins (UFT) e ao Conselho Nacional de Desenvolvimento Cientifico Tecnológico (CNPq) pela bolsa concedida.

Todos os autores declararam não haver qualquer potencial conflito de interesses referente a este artigo.

\section{REFERÊNCIAS}

ALVARÃES, A. de O.; GONÇALVES, I. C.; PEIXOTO, L. B. Análise termodinâmica da produção de 5-hidroximetilfurfural (HMF) a partir da glicose, Universidade Federal
ANEEL, 2012, Banco de Informações de Geração. Disponível em: 〈www.aneel.gov.br>. Acesso em: 09/02/2021.

AZEVEDO, V. Q. Caracterização e rendimento de hidrólise e fermentação de biomassa de arundo, palha e casca de arroz. 2015. 49 f. TCC - Curso de Engenharia Ambiental e Sanitária, Centro de Engenharias, Universidade Federal de Pelotas, Pelotas, 2015.

BASTOS, Valéria D. "Biopolímeros e polímeros de matérias-primas renováveis". Revista do BNDES, v. 14, n. 28, p. 201-234, BNDES, Rio de Janeiro, dez. 2007.

BEVILAQUA, D. B. Produção de ácido levulínico por meio de hidrólise ácida da casca de arroz. Dissertação de Mestrado, Universidade Federal de Santa Maria, Santa Maria - RS, Brasil, 87, 2010.

BRAGA, R. M., FONTES, M. S. B., AQUINO, F. M., MELO, D. M. A., MELO, M. A. F., FREITAS, J. C. O., \& BARROS, J. Estudo Comparativo da Cinética da Pirólise do Capim 
Elefante e da Casca do Arroz. In VIII Congresso Brasileiro de Análise Térmica e Calorimetria e III Congresso Pan-Americano de Análise Térmica e Calorimatria. 2012.

BRUUN, S.; JENSEN, J. W.; MAGID, J.; LINDEDAM, J.; ENGELSEN, S. B. Prediction of the degradability and ash content of wheat straw from different cultivars using near infrared spectroscopy. Industrial Crops And Products, 2010, [s.1.], v. 31, n. 2, p.321-326.

CORTEZ, Luís Augusto Barbosa, LORA, Electo Eduardo Silva, GÓMEZ, Edgardo Olivares, 2008, Biomassa para Energia. 1 ed. Campinas, Editora da Unicamp.

DE CARVALHO, E. G., RODRIGUES, F. D. A., MONTEIRO, R. S., RIBAS, R. M., \& DA SILVA, M. J. Experimental design and economic analysis of 5-hydroxymethylfurfural synthesis from fructose in acetone-water system using niobium phosphate as catalyst. Biomass Conversion and Biorefinery, 2018, 8(3), 635646.

DHARASKAR, S. A.; VARMA, M. N.; SHENDE, D. Z.; YOO, C. Y.; KAILAS, L.; WASEWAR, K. L. Synthesis, Characterization and Application of 1-Butyl-3 Methylimidazolium Chloride as Green Material for Extractive Desulfurization of Liquid Fuel. The Scientific World Journal, 2013, [s.1.], v. 2013, p.1-9.

DUNNING, J.W.; DALLAS, D. E. Analytical procedures for control of saccharification operations. Analytical Chemistry, 1949, v.21, n. 6, p.727-729.

FOLETTO, E. L., Hoffmann, R., Hoffmann, R. S., Portugal Jr, U. L., \& Jahn, S. L. (2005). Aplicabilidade das cinzas da casca de arroz. Química Nova, 28(6), 1055-1060.

HU, L.; YANG, S. Catalytic Transformation of Fructose and Sucrose to HMF with ProlineDerived Ionic Liquids under Mild Conditions. International Journal of Chemical Engineering, 2014, v. 2014, Article ID 978708, 7 pages.

IBGE, Instituto Brasileiro de Geografia e Estatística, Disponível em: http://www.ibge.gov.br/home/, 2020.
IRIS, K. M., TSANG, D. C., YIP, A. C., CHEN, S. S., WANG, L., OK, Y. S., \& POON, C. S. Catalytic valorization of starch-rich food waste into hydroxymethylfurfural (HMF): controlling relative kinetics for high productivity. Bioresource technology, 2017, 237: 222-230.

KLÄUSLI, T.. AVA Biochem: commercialising renewable platform chemical 5-HMF. Green Processing and Synthesis, 2014, 3.3: 235-236.

KLAUTAU, J. V. P. Análise Experimental de uma Fornalha a lenha de Fluxo Cocorrente Para Secagem de Grãos. Dissertação mestrado em Engenharia de Recursos Hídricos e Ambiental, PPGERHA, UFPR. Curitiba: 2008.

LI, M., LI, W., LU, Y., JAMEEL, H., CHANG, H. M., \& MA, L. High conversion of glucose to 5-hydroxymethylfurfural using hydrochloric acid as a catalyst and sodium chloride as a promoter in a water $/ \gamma$-valerolactone system. $\quad \boldsymbol{R S C}$ advances, 2017, 7(24), 14330-14336.

OLIVIER-BOURBIGOU, H.; MAGNA, L.; MORVAN, D. Ionic liquids and catalysis: Recent progress from knowledge to applications. Applied Catalysis A: General, v. 315 373 , n. 1-2, p. 1-56, 2010.

PELETEIRO, S. RIVAS, S.; ALONSO, J. A.; VALENTIN. S.; PARAJÓ, J. C. Furfural production using ionic liquids: A review. Bioresource Technology, 2016, [s.1.], v. 202, p.181-191.

POUEY, M. T. F. Beneficiamento da cinza da casca de arroz residual com vistas à produção de cimento e/ou composto pozolânico. Tese de doutorado, UFRGS, 2006.

RAMBO, M. K. D., ALMEIDA, K. J. C. R. , RAMBO, M. C. D., \& BARUQUE FILHO, E. A. The Response Surface Methodology as a tool to optimize the extraction and acid hydrolysis processes applied to babassu residues. $R B P G$. Revista Brasileira de Pós-Graduação, 2016, 13(32).

Rocha, G. J. M.; Silva, J. S.; Resumos do XVI Congresso Brasileiro de Engenharia Química, São Paulo, Brasil, 2006.

ROSATELlA, A. A., SIMEONOV, S. P., FRADE, R. F., \& AFONSO, C. A. 5Hydroxymethylfurfural (HMF) as a building 
block platform: Biological properties, synthesis and synthetic applications. Green Chemistry, 2011, 13(4), 754-793.

SCAPIN, E.; RAMBO, M. K. D.; VIANA, G. C. C.; MARASCA, N.; LACERDA, G. E.; RAMBO, M. C. D.; FERNANDES, R. M. N.; Sustainable production of furfural and 5-hidroximetilfurfural from rice husks and soybean peel by using ionic liquid. Food Science And Technology, 2020, [s.l.], DOI: 10.1590/fst.04419.

SCAPIN, E.; RAMBO, M. K. D.; VIANA, G. C. C.; BORGES, M. S.; RAMBO, M. C. D.; CARNEIRO, C. Production of Furanic Compounds and Organic Acids from Brazilian Pequi (Caryocar brasiliensis Camb.) Residues Using Green Chemistry. Journal of The Brazilian Chemical Society, [s.1.], 2020. DOI: 10.21577/0103-5053.20200023.

STARK, A. Ionic liquids in the biorefinery: a critical assessment of their potential. Energy \& Environmental Science, v. 4, n. 1, p. 19-32, 2011.

TRUJILlO, A. I., MARICHAL, M. D. J., \& CARRIQUIRY, M. Comparison of dry matter and neutral detergent fibre degradation of fibrous feedstuffs as determined with in situ and in vitro gravimetric procedures. Animal feed science and technology, 2010, 161(1-2), 49-57.

VAN DAM, J.; GOSSELINK, R.; JONG, E. Lignin Applications. Agrotechnology e Food Innovations. Disponivel em $<\mathrm{http} / /$ www.biomassandbioenergy.nl>. Acesso em 04 març. 2020.

VAN NGUYEN, C., LEWIS, D., CHEN, W. H., HUANG, H. W., ALOTHMAN, Z. A., YAMAUCHI, Y., \& WU, K. C. W. Combined treatments for producing 5hydroxymethylfurfural (HMF) from lignocellulosic biomass. Catalysis Today, 2016, 278, 344-349.

VAN Putten, R. J., Van Der Waal, J. C., De Jong, E. D., Rasrendra, C. B., Heeres, H. J., \& de Vries, J. G. (2013). Hydroxymethylfurfural, a versatile platform chemical made from renewable resources. Chemical reviews, 113(3), 1499-1597. JEFFRIES, T. W. Utilization of xylose by actéria, yeast and fungi. Advances in Biochemical Engineering, v. 27, p. 1-32,1983.
VIEIRA, A. C., DE SOUZA, S. N. M., BARICCATTI, R. A., SIQUEIRA, J. A. C., \& NOGUEIRA, C. E. C. Caracterização da casca de arroz para geração de energia. Varia Scientia Agrárias, 2013, 3(1), 51-57.

WANG H.; Z., D.; LI, Q.; LIU, J.; TAN, C.; WANG, C.; CAI, L. M. Recent advances in catalytic conversion of biomass to 5hydroxymethylfurfural and 2,5- dimethylfuran, Renew. Sustain. Energy Rev. 2019, 103, p. 227 247.

WANG, S., DU, Y., ZHANG, P., CHENG, X., \& QU, Y. One-pot synthesis of 5hydroxymethylfurfural directly from cottonseed hull biomass using chromium (III) chloride in ionic liquid. Korean Journal of Chemical Engineering, 2014, 31.12: 2286-2290.

YANG, Y.; HU, C.-w.; ABU-OMAR, M. M.. Conversion of carbohydrates and lignocellulosic biomass into 5-hydroxymethylfurfural using $\mathrm{AlCl}_{3} \cdot 6 \mathrm{H}_{2} \mathrm{O}$ catalyst in a biphasic solvent system. Green Chem., 2012, [s.1.], v. 14, n. 2, p.509-513.

YASSIN, F. A. Highly effective ionic liquids for biodiesel production from waste vegetable oils. Egyptian Journal Of Petroleum, 2015, v. 24, n. 316 1, p.103-111.

YI, Y. B. HA, M. G. LEE, J. W. CHUNG

C. H. New role of choromium fluoride: Its catalytic action on the synthesis of hydroxymethylfurfural in ionic liquid using raw plant biomass and characterization of biomass hydrolysis. Chemical Engineering Journal. Busan, South Korea, p. 370375. 19 out. 2011.

YU, I.K.M.; TSANG, D.C.W.; YIP, A.C.K.; CHEN S.S.; OK, Y.S.; POON C.S. 2017. Catalytic valorization of starch-rich food waste into hydroxymethylfurfural (HMF): controlling relative kinetics for high productivity. Bioresource Technology. 237, 222-230.

ZHANG, L., XI, G., ZHANG, J., YU, H., \& WANG, X. Efficient catalytic system for the direct transformation of lignocellulosic biomass to furfural and 5hydroxymethylfurfural. Bioresource technology, 2017, 224, 656-661.

ZHANG, Z.; ZHAO, Z. K.. Microwave-assisted conversion of lignocellulosic biomass into furans 
in ionic liquid. Bioresource Technology, 2010,

[s.1.], v. 101, n. 3, p.1111-1114. 